\title{
When structures are almost surely connected
}

\author{
Jason P. Bell \\ Department of Mathematics \\ University of California San Diego \\ La Jolla, CA 92093-0112. USA \\ jbell@math.ucsd.edu
}

Submitted: June 5, 2000

Accepted: July 20, 2000

\begin{abstract}
Let $A_{n}$ denote the number of objects of some type of "size" $n$, and let $C_{n}$ denote the number of these objects which are connected. It is often the case that there is a relation between a generating function of the $C_{n}$ 's and a generating function of the $A_{n}$ 's. Wright showed that if $\lim _{n \rightarrow \infty} C_{n} / A_{n}=1$, then the radius of convergence of these generating functions must be zero. In this paper we prove that if the radius of convergence of the generating functions is zero, then $\lim \sup _{n \rightarrow \infty} C_{n} / A_{n}=1$, proving a conjecture of Compton; moreover, we show that $\liminf \operatorname{in}_{n \rightarrow \infty} C_{n} / A_{n}$ can assume any value between 0 and 1 .
\end{abstract}

\section{Introduction}

Let $A_{n}$ count objects of some type by their "size" $n$ and let $C_{n}$ count those which are connected. One frequently has either

$$
A(x)=\exp (C(x)) \quad \text { or } \quad A(x)=\exp \left(\sum_{k \geq 1} \frac{C\left(x^{k}\right)}{k}\right),
$$

for exponential generating functions of labeled objects and ordinary generating functions of unlabeled objects, respectively. Let $R$ be the radius of convergence of the power series. Various authors have studied the limiting behavior of $C_{n} / A_{n}$. In particular, Wright [3] constructed a sequence $\left\{C_{n}\right\}_{n \geq 1}$ such that $\lim \sup C_{n} / A_{n}=1$ and $\lim \inf C_{n} / A_{n}<2 / 3$ in both the labeled and unlabeled case. Also, Wright [3], [4] showed that if $\lim _{n \rightarrow \infty} C_{n} / A_{n}=1$, then $R=0$. Compton [1] asked if the converse were true, assuming the limit exists. The following theorem provides an affirmative answer.

Theorem 1 Suppose that either of (1.1) holds then:

- If $R=0$, then $\limsup _{n \rightarrow \infty} C_{n} / A_{n}=1$. 
THE ELECTRONiC JOURNAL of COMBinAtorics 7 (2000), \#R36

- For any $0 \leq l \leq 1$, there exists both labeled and unlabeled objects satisfying (1.1) with $R=0$ and $\liminf _{n \rightarrow \infty} C_{n} / A_{n}=l$.

Combining the first part of the theorem with Wright's result shows that, if $\lim _{n \rightarrow \infty} C_{n} / A_{n}=\rho$ exists, then $\rho=1$ if and only if $R=0$.

The author would like to thank Ed Bender for helpful advice about the exposition of this paper.

\section{Proofs}

We require the following simple lemma.

Lemma 1 Suppose $p(x)=\sum_{i=1}^{\infty} p_{i} x^{i}\left(p_{1} \neq 0\right)$ is analytic at zero and suppose $h(x)=$ $\sum_{i=1}^{\infty} h_{i} x^{i}$ has the property that $p(h(x))=g(x)$ is a power series that is analytic at zero. Then $h(x)$ is analytic at zero.

Proof. Let $p^{-1}(x)$ be the formal inverse of $p$. Since $p(x)$ is analytic at zero, we have that $p^{-1}(x)$ is analytic at zero by [2] page 87, Theorem 4.5.1. Hence $h(x)=p^{-1}(g(x))$ is analytic at zero as required.

We now prove a lemma that will be useful to us.

Lemma 2 Suppose $C(x)=\sum_{i=1}^{\infty} c_{i} x^{i}$ is a power series with non-negative coefficients and

$$
p(x)=\sum_{i=1}^{\infty} p_{i} x^{i} \quad\left(p_{1} \neq 0\right)
$$

is a power series that is analytic at zero satisfying

$$
p_{n}+\alpha c_{n} \leq\left[x^{n}\right] e^{C(x)}
$$

for some $\alpha>1$ and all $n \geq 1$. Then $C(x)$ is analytic at zero.

Proof. To prove this, let us first note that if $D(x)=\sum_{i=1}^{\infty} d_{i} x^{i}$ is a formal power series that satisfies the equation

$$
p_{n}+\alpha d_{n}=\left[x^{n}\right] e^{D(x)}
$$


for all $n \geq 1$, then $D(x)$ is analytic. To see this, let us note that equation (2.2) is equivalent to stating that

$$
1+p(x)+\alpha D(x)=e^{D(x)}
$$

as formal power series. Notice that $d_{1}=-p_{1} /(\alpha-1) \neq 0$ and hence $D(x)$ has a formal inverse $D^{-1}(x)$. Substituting $x=D^{-1}(u)$ into the equation (2.3), we find that

$$
p\left(D^{-1}(u)\right)=e^{u}-\alpha u-1 .
$$

Thus by Lemma 1 we have that $D^{-1}(u)$ is analytic at zero. By Lemma 1 we have that $D(x)$ is analytic at zero. We now show that $0 \leq c_{n} \leq d_{n}$ for all $n \geq 1$. We prove this by induction on $n$. Note that for $n=1$, we have that $p_{1}+\alpha c_{1} \leq[x] e^{C(x)}=c_{1}$ and so $c_{1} \leq-p_{1} /(\alpha-1)=d_{1}$. Hence the claim is true when $n=1$. Suppose the claim is true for all values less than $n$. We have

$$
\begin{aligned}
p_{n}+\alpha c_{n} & \leq\left[x^{n}\right] e^{C(x)} \\
& =\left[x^{n}\right] \exp \left(c_{1} x+c_{2} x^{2}+\cdots+c_{n} x^{n}\right) \\
& \leq\left[x^{n}\right] \exp \left(d_{1} x+d_{2} x^{2}+\cdots+d_{n} x^{n}+\left(c_{n}-d_{n}\right) x^{n}\right)
\end{aligned}
$$

since $c_{k} \leq d_{k}$ for $k<n$. Thus

$$
\begin{aligned}
p_{n}+\alpha c_{n} & \leq\left[x^{n}\right] \exp \left(d_{1} x+\cdots+d_{n} x^{n}\right) \exp \left(\left(c_{n}-d_{n}\right) x^{n}\right) \\
& =\left[x^{n}\right] \exp (D(x))\left(1+\left(c_{n}-d_{n}\right) x^{n}\right) \\
& =\left[x^{n}\right] \exp (D(x))+c_{n}-d_{n} \\
& =p_{n}+\alpha d_{n}+c_{n}-d_{n} .
\end{aligned}
$$

Hence $(\alpha-1) c_{n} \leq(\alpha-1) d_{n}$ and so $0 \leq c_{n} \leq d_{n}$ for all $n \geq 1$. Since $D(x)$ is analytic at zero, it follows that $C(x)$ is analytic at zero. This completes the proof.

The following theorem implies the first part of Theorem 1. To see this, it suffices to note that

$$
\left[x^{n}\right] \exp (C(x)) \leq\left[x^{n}\right] \exp \left(\sum_{k \geq 1} \frac{C\left(x^{k}\right)}{k}\right) .
$$

Theorem 2 Suppose $c_{i} \geq 0$ for all $i$ and $C(x)=\sum_{i=1}^{\infty} c_{i} x^{i}$ has radius of convergence zero. Let

$$
A(x)=\sum_{i=1}^{\infty} a_{i} x^{i}=\exp \left(\sum_{j=1}^{\infty} C\left(x^{j}\right) / j\right) .
$$

Then

$$
\limsup _{n \rightarrow \infty} \frac{c_{n}}{a_{n}}=1
$$


Proof. Without loss of generality we may assume that $c_{1} \geq 1$, as increasing the value of $c_{1}$ can only decrease the values of $c_{n} / a_{n}$ for large $n$. Suppose

$$
\limsup _{n \rightarrow \infty} \frac{c_{n}}{a_{n}} \neq 1
$$

Then there exists $\lambda>1$ and a positive integer $N$ such that

$$
\frac{a_{n}}{c_{n}}>\lambda \quad \text { for all } n>N
$$

Let $H(x)=\sum_{i=1}^{\infty} h_{i} x^{i}$ be the power series

$$
H(x)=\sum_{k=1}^{\infty} \frac{C\left(x^{k}\right)}{k} \quad \text { so that } \quad c_{n}=\sum_{d \mid n} \frac{\mu(d) h_{n / d}}{d} .
$$

Define the two sets

$$
S_{1}=\left\{\begin{array}{l|l}
n>N & \frac{a_{n}}{h_{n}} \geq \frac{1+\lambda}{2}
\end{array}\right\}
$$

and

$$
S_{2}=\left\{\begin{array}{l|l}
n>N & \frac{a_{n}}{h_{n}}<\frac{1+\lambda}{2}
\end{array}\right\} .
$$

If $n \in S_{2}$, then by (2.4) we must have that $c_{n} / h_{n}<(1+\lambda) / 2 \lambda$. Thus

$$
\sum_{d \mid n} \frac{\mu(d) h_{n / d}}{d}<\frac{(1+\lambda) h_{n}}{2 \lambda}
$$

But

$$
\begin{aligned}
\sum_{d \mid n} \frac{\mu(d) h_{n / d}}{d} & =h_{n}+\sum_{\substack{d \mid n \\
d \neq 1}} \frac{\mu(d) h_{n / d}}{d} \\
& \geq h_{n}-\sum_{\substack{d \mid n \\
d \neq 1}} \frac{h_{n / d}}{d} .
\end{aligned}
$$

Combining this result with (2.7) we find that there exists some divisor $d \neq 1$ of $n$ such that $h_{n / d} / d>(\lambda-1) h_{n} / 2 d(n) \lambda$. Hence

$$
\begin{aligned}
h_{n}(1+\lambda) / 2>a_{n} & =\left[x^{n}\right] e^{H(x)} \\
& \geq h_{n}+h_{n / d}^{d} / d ! \\
& \geq h_{n}+\frac{\left((\lambda-1) d h_{n}\right)^{d}}{(2 d(n) \lambda)^{d} d !} \\
& \geq h_{n}+\frac{(\lambda-1)^{d} h_{n}^{d}}{(2 n \lambda)^{d}} .
\end{aligned}
$$


Solving for $h_{n}$ we find that

$$
h_{n}<\left(\frac{2 n \lambda\left(\frac{\lambda-1}{2}\right)^{1 / d}}{\lambda-1}\right)^{d /(d-1)}=O\left(n^{2}\right)
$$

and so there exists $C>0$ such that $h_{n}<C n^{2}$ for all $n \in S_{2} \cup\{1,2, \ldots, N\}$; that is, all $n \notin S_{1}$. Define

$$
p(x)=-\frac{(1+\lambda)}{2}\left(\sum_{j=1}^{N} C j^{2} x^{j}+\sum_{j \in S_{2}} C j^{2} x^{j}\right) .
$$

Clearly $p(x)$ has a radius of convergence of at least 1 and so it is analytic at zero. Consider the power series $p(x)+(1+\lambda) H(x) / 2$. Notice if $n \notin S_{1}$, then

$$
\begin{aligned}
{\left[x^{n}\right]\left(p(x)+\frac{(1+\lambda)}{2} H(x)\right) } & =\frac{(1+\lambda)}{2}\left(-C n^{2}+h_{n}\right) \\
& \leq 0 \\
& \leq a_{n} \\
& =\left[x^{n}\right] \exp (H(x)) .
\end{aligned}
$$

If $n \in S_{1}$, then

$$
\left[x^{n}\right]\left(p(x)+\frac{(1+\lambda)}{2} H(x)\right)=(1+\lambda) h_{n} / 2 \leq a_{n}=\left[x^{n}\right] \exp (H(x)) .
$$

Hence we have

$$
\left[x^{n}\right]\left(p(x)+\frac{(1+\lambda)}{2} H(x)\right) \leq\left[x^{n}\right] \exp (H(x))
$$

for all $n \geq 1$. Moreover when $n=1, p^{\prime}(0)+\frac{1+\lambda}{2} h_{1} \leq h_{1}$, and so $p^{\prime}(0)<0$. Hence by Lemma 2, $H(x)$ is analytic at zero. Since $0 \leq c_{n} \leq h_{n}$ for all $n$, we see that $C(x)$ is also analytic at zero, a contradiction. This completes the proof of the theorem.

We now prove the second part of Theorem 1. The set of all graphs (labeled or unlabeled) provides an example for $l=1$ [5]. For $l=0$, notice if $C(x)=\sum_{n \geq 1} C_{n} x^{n}$ is any power series of radius zero having positive integer coefficients and $C_{n}=1$ for infinitely many $n$, then in both the labeled and unlabeled cases we have that

$$
\begin{aligned}
A_{n} & \geq\left[x^{n}\right] \exp (C(x)) \\
& \geq\left[x^{n}\right] \exp \left(\frac{x}{1-x}\right) \\
& \geq\left[x^{n}\right] \frac{1}{2 !} \frac{x^{2}}{(1-x)^{2}} \\
& =(n-1) / 2 .
\end{aligned}
$$


Hence

$$
\inf _{\left\{n: C_{n}=1\right\}} C_{n} / A_{n}=0 .
$$

Hence to prove the second part of Theorem 1 it suffices to prove the following theorem.

Theorem 3 Given $l$ with $0<l<1$, there exist power series $C(x)=\sum_{i \geq 1} c_{i} x^{i}$, $H(x)=\sum_{i \geq 1} h_{i} x^{i}$, and $A(x)=\sum_{i \geq 1} a_{i} x^{i}$ that satisfy the following:

1. $C(x), H(x)$, and $A(x)$ all have zero radius of convergence;

2. $c_{n}, a_{n}$, and $n ! h_{n}$ are positive integers;

3. $A(x)=\exp (H(x))=\exp \left(\sum_{j \geq 1} C\left(x^{j}\right) / j\right)$;

4. $\liminf _{n \rightarrow \infty} c_{n} / a_{n}=\liminf _{n \rightarrow \infty} h_{n} / a_{n}=l$.

Proof. We recursively define sequences $\left\{N_{n}\right\}$, and $\left\{c_{n}\right\}$ as follows. We define $N_{1}=0$, and $c_{1}=1$. For $n>1$, we define $N_{n}=\left[x^{n}\right] \prod_{j=1}^{n-1}\left(1-x^{j}\right)^{-c_{j}}$ and

$$
c_{n}= \begin{cases}n ! N_{n} & \text { if } n \text { is even } \\ {\left[\frac{N_{n}}{\alpha-1}\right]+1} & \text { if } n \text { is odd }\end{cases}
$$

where $\alpha=1 / l$. Notice $N_{n}$ and $c_{n}$ are positive integers for all $n>1$. Notice that if $n$ is even, then $c_{n} \geq n$ ! and so $C(x)$ has zero radius of convergence. Since

$$
\begin{aligned}
{\left[x^{n}\right] \prod_{j=1}^{\infty}\left(1-x^{j}\right)^{-c_{j}} } & =\left[x^{n}\right]\left(1+c_{n} x^{n}\right) \prod_{j=1}^{n-1}\left(1-x^{j}\right)^{-c_{j}} \\
& =c_{n}+N_{n} \\
& =c_{n}\left(1+N_{n} / c_{n}\right)
\end{aligned}
$$

we have that

$$
1+\sum_{j=1}^{\infty}\left(1+N_{j} / c_{j}\right) c_{j} x^{j}=\prod_{j=1}^{\infty}\left(1-x^{j}\right)^{-c_{j}}
$$

and so

$$
1+\sum_{j=1}^{\infty}\left(1+N_{j} / c_{j}\right) c_{j} x^{j}=\exp \left(\sum_{k=1}^{\infty} C\left(x^{k}\right) / k\right) .
$$

Hence $a_{n}=\left(1+N_{n} / c_{n}\right) c_{n}$. Notice that

$$
\begin{aligned}
N_{n} & =\left[x^{n}\right] \prod_{j=1}^{n-1}\left(1-x^{j}\right)^{-c_{j}} \\
& \geq\left[x^{n}\right] \prod_{j=1}^{n-1}\left(1-x^{j}\right)^{-1} \\
& \geq p(n-1) .
\end{aligned}
$$


Hence $N_{n}$ tends to infinity as $n$ tends to infinity, and so for odd $n$ we have

$$
a_{n} / c_{n}=1+\frac{N_{n}}{\left[N_{n} /(\alpha-1)\right]+1} \rightarrow \alpha
$$

as $n$ tends to infinity. Moreover, we have that for $n$ even, $a_{n} / c_{n}=1+1 / n ! \rightarrow 1$ as $n \rightarrow \infty$. Thus $C(x) \in \mathbb{Z}[[x]]$ is a power series satisfying the conditions of the theorem.

Since $H(x)=\sum_{j=1}^{\infty} C\left(x^{j}\right) / j$, we have that $h_{n}=\sum_{d \mid n} c_{n / d} / d$. Clearly $n ! h_{n}$ is a positive integer for all $n \geq 1$. To complete the proof of the theorem, it suffices to show that $\lim _{n \rightarrow \infty} h_{n} / c_{n}=1$. To see this, notice that if $n>2$, then

$$
N_{n}=\left[x^{n}\right] \prod_{j=1}^{n-1}\left(1-x^{j}\right)^{-c_{j}} \geq(1+x)^{c_{1}}\left(1+x^{n-1}\right)^{c_{n-1}}=c_{n-1} c_{1} .
$$

Since $c_{1}=1, N_{n} \geq c_{n-1}$ for all $n>1$. Thus $c_{n} \geq n ! c_{n-1}$ for even $n$ and $c_{n} \geq$ $c_{n-1} /(\alpha-1)$ for odd $n$. It follows that $c_{n} \geq(n-1) ! c_{n-2} /(\alpha-1)$ for all $n>2$, and so there is a $B>0$ such that $c_{n} \geq B(n-1) ! c_{k}$ for all $k \leq n / 2$. Hence we have that for $n>2$

$$
\begin{aligned}
h_{n} & =c_{n}+\sum_{\substack{d \mid n \\
d \neq 1}} c_{n / d} / d \\
& \leq c_{n}\left(1+\sum_{\substack{d \mid n \\
d \neq 1}} 1 / B(n-1) !\right) \\
& =c_{n}(1+o(1)) .
\end{aligned}
$$

This completes the proof of the theorem.

\section{References}

[1] K. J. Compton, Some methods for computing component distribution probabilities in relational structures, Discrete Math. 66 (1987) 59-77.

[2] E. Hille, Analytic function theory. Vol. I. Introduction to Higher Mathematics Ginn and Company, Boston (1959).

[3] E. M. Wright, A relationship between two sequences, Proc. London Math. Soc. (iii) 17 (1967) 296-304.

[4] E. M. Wright, A relationship between two sequences III, J. London Math. Soc. 43 (1968) 720-724.

[5] E. M. Wright, Asymptotic relations between enumerative functions in graph theory, Proc. London Math. Soc. (iii) 20 (1970) 558-572. 